(C) 2003 International Press

Adv. Theor. Math. Phys. 1 (2003) 1-23

\title{
A note on E-strings
}

\author{
Amer Iqbal \\ Theory Group, Department of Physics \\ University of Texas at Austin, \\ Austin, TX 78712, U.S.A. \\ iqbal@physics.utexas.edu
}

\begin{abstract}
We study BPS states in type IIA string compactification on a local Calabi-Yau 3-fold which are related to the BPS states of the E-string. Using Picard-Lefshetz transformations of the 3-cycles on the mirror manifold we determine automorphisms of the K-theory of the compact divisor of the Calabi-Yau which maps certain D-brane configurations to a bound state of single D4-brane with multiple D0-branes. This map allows us to write down the generating functions for the multiplicity of these BPS states.
\end{abstract}

e-print archive: http://lanl.arXiv.org/abs/hep-th/0206064 


\section{Introduction}

String dualities have played an important role in our understanding of perturbative and non-perturbative phenomena in supersymmetric theories. An interesting and important application of dualities has been to the enumerative geometry of maps. BPS amplitudes in string theory which are protected by supersymmetry from quantum corrections have interesting mathematical interpretations in the dual theory. Notable among these are the $\mathcal{N}=2$ topological string partition functions which count curves of various genera $[1,2,3], \mathcal{N}=1$ superpotential which counts maps from a disk with boundary mapped to a Lagrangian cycles $[4,5,6,7,8,9,10]$ and the partition function of the $\mathcal{N}=4$ Vafa-Witten theory which is the generating function of the Euler characteristic of the instanton moduli spaces $[11,12,13,14,15,16,17,18,19,20,21,22,23]$.

In this paper we will discuss the partition functions of the Vafa-Witten (VW) theory and its relation with the E-string [12, 24, 25, 26] partition functions [12]. String dualities allow us to interpret the partition function of the VW theory as the generating function for the multiplicities of certain BPS states in the type IIA string compactification on a Calabi-Yau threefold. These partition functions have been evaluated for only a few cases such as $T^{4}, K 3$ and the rational elliptic surface $\mathcal{B}_{9}\left(\mathbb{P}^{2} \text { blown up at nine points }\right)^{1}$. In the case of $\mathcal{B}_{9}$ the expressions obtained in [12], partition functions of E-string, were a sum, over the flux in the fiber direction, of the partition functions $Z_{\Sigma}^{r}(\tau)$ of the VW theory,

$$
\mathcal{Z}_{r, \Sigma}(\tau)=\frac{1}{r} \sum_{n=0}^{r-1} Z_{\Sigma+n F}^{r}(\tau) .
$$

Where $F$ is the class of the fiber and $\Sigma \in H_{2}\left(\mathcal{B}_{9}, \mathbb{Z}\right)$ is such that $\Sigma \cdot F=0$. The purpose of this note is to show that Picard-Lefshetz transformations of the 3-cycles in the mirror manifold $Y$ and the map from $H_{*}(X)$ to $H_{3}(Y)$ allows us to map an arbitrary D-brane configuration to a bound state of D2-brane and D0-branes. Therefore allowing us to compute $Z_{\Sigma}^{r}(\tau)$ without any restriction on $\Sigma \cdot F$.

The configurations studied in [12] were bound states of $Q_{4} \mathrm{D} 4$-branes wrapped on $\mathcal{B}_{9}$ and $Q_{0}$ D0-branes. Since $\mathcal{B}_{9}$ is an elliptically fibered manifold therefore T-duality on the elliptic curve converts the D4-brane into a D2-brane

\footnotetext{
${ }^{1}$ In [11] a conjecture for the rank two partition functions of the VW theory on $\mathbb{P}^{2}$ was also given. Also a conjecture about the $U(r)$ partition function ( $r$ an odd prime) of VW theory on $\mathcal{B}_{9}$ was made in [23]
} 
wrapped on the base of the fibration and converts the D0-brane into D2brane wrapped on the elliptic fiber. Thus the D4-brane/D0-brane configuration is mapped to a D2-brane wrapping a two cycle in $\mathcal{B}_{9}$. Thus the problem of counting the multiplicity of the BPS states given by D4-brane/D0-brane bound states becomes the problem of counting certain curves in Calabi-Yau threefold $X$ which can be done using mirror symmetry. The configuration of D4-brane/D0-brane we started with, however, is not the most general possible BPS configuration since one can also have D2-brane wrapped on some curve. Such a configuration with D2-brane wrapped on a curve, however, cannot be mapped to a D2-brane wrapping some other curve using the T-duality on the fiber of $\mathcal{B}_{9}$. We will show that since we can perform $S L(2, \mathbb{Z})$ transformations on the fiber we can map an arbitrary configuration of D4/D2/D0-branes to a D2-brane wrapped on a curve with some D0-branes.

The paper is organized as follows. In section two we review some facts about the second homology lattice of $\mathcal{B}_{9}$, the structure of the curve counting functions, and their relation with E-strings partition functions and partition functions of VW theory. In section three we discuss the action of $S L(2, \mathbb{Z})$ and the generalized Weyl transformations on the D-brane configurations. In section four we use the transformations discussed in section three to write down partition functions of VW theory. Some facts about the curves in $\mathcal{B}_{9}$ and the action of affine $E_{8}$ on the curves is discussed in appendix A.

After this paper appeared we were informed by Kota Yoshioka that theorem 6.15 and corollary 6.18 of his paper "Twisted stability and Fourier-Mukai Transform" (math.AG/0106118) may provide mathematical evidence for the results of section three.

\section{Review of curve counting and E-string partition functions}

In this section we will review the relation between the curve counting functions (genus zero topological string amplitude) and the E-string partition functions [12].

The Calabi-Yau space we will consider is total space of the canonical line bundle over the rational elliptic surface. We will denote the CY space by $X$ and the compact divisor by $\mathcal{B}_{9}$. $\mathcal{B}_{9}$ is an elliptically fibered surface with twelve degenerate fibers on the base $\mathbb{P}^{1}$. For this reason it is also known as 
$\frac{1}{2} \mathrm{~K} 3$. For a more detailed construction of this CY from an orbifold see [12].

\section{$2.1 \quad H_{2}\left(\mathcal{B}_{9}\right)$ and affine $E_{8}$}

The compact divisor $\mathcal{B}_{9}$ can be obtained from $\mathbb{P}^{2}$ by blown up nine points. We denote by $\left\{h, e_{1}, \cdots, e_{9}\right\}$ a basis of $H_{2}\left(\mathcal{B}_{9}, \mathbb{Z}\right)$ such that

$$
h^{2}=1, h \cdot e_{i}=0, e_{i} \cdot e_{j}=-\delta_{i j} .
$$

Here $h$ is the pullback of the generator of $\mathbb{P}^{2}$ and $e_{i}$ are the exceptional curves introduced by blowing up $\mathbb{P}^{2}$ at nine points. The canonical class is given by $3 h-\sum_{i=1}^{9} e_{i}$ and the homology classes orthogonal to it forms a codimension one lattice isomorphic to the affine $E_{8}$ lattice. The real simple roots $\left\{\alpha_{1}, \cdots, \alpha_{8}\right\}$ and the imaginary root $F$ of this affine $E_{8}$ are

$$
\begin{aligned}
\alpha_{i} & :=e_{i}-e_{i+1}, i=1, \cdots, 7, \\
\alpha_{8} & :=h-e_{1}-e_{2}-e_{3}, \\
F & :=3 h-\sum_{i=1}^{9} e_{i} .
\end{aligned}
$$

From eq(2) it follows that the imaginary root and the simple roots defined above have the following intersection numbers,

$$
\alpha_{a} \cdot \alpha_{b}=-\left(A_{E_{8}}\right)_{a b}, \alpha_{a} \cdot F=0, F^{2}=0, a=1, \cdots 8 .
$$

Where $A_{E_{8}}$ is the $E_{8}$ Cartan matrix. It is convenient to define a different basis $\left\{\omega^{1}, \cdots, \omega^{8}, B, F\right\}$ of $H_{2}\left(\mathcal{B}_{9}\right)$ such that,

$$
\omega^{a}:=\sum_{b=1}^{8}\left(A_{E_{8}}^{-1}\right)_{a b} \alpha_{b}, B:=e_{9} .
$$

The classes $B$ and $F$ are the homology classes of the base $\mathbb{P}^{1}$ and fiber $T^{2}$ respectively and satisfy,

$$
\begin{aligned}
B^{2} & =-1, F^{2}=0, B \cdot F=1, \\
B \cdot \omega^{a} & =F \cdot \omega^{a}=0, a=1, \cdots, 8 .
\end{aligned}
$$

In this basis we can write any class $\Sigma \in H_{2}\left(\mathcal{B}_{9}, \mathbb{Z}\right)$ as,

$$
\Sigma=\sum_{a=1}^{8} \lambda_{a} \omega^{a}+d B+s F, \text { where } \lambda_{a}=\Sigma \cdot \alpha_{a}, d=\Sigma \cdot F, s=\Sigma \cdot(B+F) .
$$


We will always use this basis to represent homology classes and therefore will use the notation $(\vec{\lambda}, d, s)$ to represent the curve given above. For $\Sigma_{i}=$ $\left(\vec{\lambda}_{i}, d_{i}, s_{i}\right)$ the intersection product is given by ${ }^{2}$

$$
\Sigma_{1} \cdot \Sigma_{2}=-\overrightarrow{\lambda_{1}} \cdot \overrightarrow{\lambda_{2}}-d_{1} d_{2}+d_{1} s_{2}+d_{2} s_{1}
$$

For an effective curve $\Sigma$ the virtual genus $g$ can be determined from the Adjunction formula $\Sigma \cdot \Sigma=2 g-2+d$. The intersection of the curve with the canonical class, $d$, is called the degree of the curve.

$E_{8}$ Weyl transformations map $H_{2}\left(\mathcal{B}_{9}\right)$ to itself such that if $w \in W\left(E_{8}\right)$ and $\Sigma=(\vec{\lambda}, d, s)$ then

$$
w(\Sigma)=(w(\vec{\lambda}), d, s)
$$

Since the codimension one lattice orthogonal to the canonical class is affine $E_{8}$ lattice we also have affine $E_{8}$ Weyl transformations. These are reflections in the roots $\widehat{\alpha}_{m}=\alpha+m F$ where $\alpha$ is an $E_{8}$ root and $m \in \mathbb{Z}$. These affine Weyl transformation are such that if $\Sigma_{1}=\left(\vec{\lambda}_{1}, d_{1}, s_{1}\right)$ and $\Sigma_{2}=\left(\vec{\lambda}_{2}, d_{2}, s_{2}\right)$ are related by such a transformation then

$$
\vec{\lambda}_{2} \in w\left(\vec{\lambda}_{1}\right)+m d \Gamma_{E_{8}}, d_{1}=d_{2}=d, \Sigma_{1}^{2}=\Sigma_{2}^{2} .
$$

This implies that for a curve of fixed degree and genus we only need to consider curves with the highest weight vector for affine $E_{8}$ at level $d$. Two curves $\Sigma_{\lambda}$ and $\Sigma_{\mu}$ of degree $d$ and genus $g$, with $E_{8}$ weights $\lambda$ and $\mu$ related as $\mu=w(\lambda)+d \lambda^{\prime}$ can be transformed into each other by an affine $E_{8}$ Weyl-transformation. To see this consider two curves $\Sigma_{\lambda}=\left(\vec{\lambda}, d, l_{\lambda}\right)$ and $\Sigma_{\mu}=\left(\vec{\mu}, d, l_{\mu}\right)$ of genus $g$ and degree $d$ with $E_{8}$ weight vectors $\lambda$ and $\mu$ respectively. Since $\Sigma_{\lambda}^{2}=\Sigma_{\mu}^{2}=2 g-2+d$ therefore

$$
l_{\mu}=l_{\lambda}+\frac{1}{2 d}\left(\mu^{2}-\lambda^{2}\right)
$$

It follows from $\mu=w(\lambda)+d \lambda^{\prime}$ that one can write $\mu=w\left(\lambda+d \lambda^{\prime \prime}\right)$ for some $\lambda^{\prime \prime}=\sum s^{i} \alpha_{i}$, where $s^{i}$ are integers and $\alpha_{i}$ are the $E_{8}$ simple roots. Now perform the following affine $E_{8}$ Weyl-transformation: ${ }^{3}$

$$
w_{\left(\alpha_{8}, \bar{m}^{8}\right)} \circ w_{\left(\alpha_{8}, m^{8}\right)} \circ \ldots \circ w_{\left(\alpha_{1}, \bar{m}^{1}\right)} \circ w_{\left(\alpha_{1}, m^{1}\right)} ; \quad \text { with } \quad \bar{m}^{i}-m^{i}=s^{i},
$$

on the curve $\Sigma_{\lambda}=\left(\lambda, d, l_{\lambda}\right)$ to obtain:

$$
\left(\lambda+d \lambda^{\prime \prime}, d, l_{\lambda}+\frac{1}{2 d}\left\{\left(\lambda+d \lambda^{\prime \prime}\right)^{2}-\lambda^{2}\right\}\right)=\left(\lambda+d \lambda^{\prime \prime}, d, l_{\lambda}+\frac{1}{2 d}\left\{\mu^{2}-\lambda^{2}\right\}\right)
$$

\footnotetext{
${ }^{2} \vec{\lambda} \cdot \vec{\mu}=\sum_{a, b=1}^{8} \lambda_{a}\left(A_{E_{8}}^{-1}\right)_{a b} \mu_{b}$.

${ }^{3}(\alpha, 0, n)=\alpha+n F$, where $\alpha$ is an $E_{8}$ root and $n \in \mathbb{Z}$.
} 


$$
=\left(w^{-1}(\mu), d, l_{\mu}\right) .
$$

This differs from the curve corresponding to $\mu$ by just a Weyl transformation of $E_{8}$. As a consequence we find that it is sufficient to study curves with dominant $E_{8}$ weight vector because any other curve can be obtained from these by an affine $E_{8}$ Weyl-transformation. Also this implies that the multiplicity of a curve depends only on its degree, genus and the equivalence class of its $E_{8}$ weight vector.

\subsection{Counting curves}

Consider the generating function for the multiplicities of degree $n$ curves.

$$
\widetilde{Z}_{n}(\omega)=\sum_{C \in H_{2}\left(\mathcal{B}_{9}, \mathbf{Z}\right), C \cdot F=n} \mathcal{N}(C) e^{2 \pi i(C \cdot \omega)},
$$

where $\mathcal{N}(C)$ is the Euler characteristic of the moduli space of class $C$ if $C$ represents a holomorphic curve and $\omega$ is the Kähler form given by

$$
\omega=\sum_{a=1}^{8} m_{a} \omega^{a}+\tau(B+F)+\phi F .
$$

so that

$$
m_{a}=\omega \cdot \alpha_{a}, \tau=\omega \cdot F, \phi=\omega \cdot B .
$$

Since the degree of the curve, which is also the level of the $E_{8}$ weight vector, is invariant under the affine Weyl transformations therefore $\lambda:=w\left(\lambda^{a}\right)+n \Gamma_{E_{8}}$ $\forall \lambda \in \Gamma_{E_{8}}$. Here $\left\{\lambda^{a} \mid a=1, \cdots F(n)\right\}$ is the set of dominant weights at level $n$. We denote by $\Delta^{a}$ the set of vectors in $\Gamma_{E_{8}}$ related to $\lambda^{a}$ by $E_{8}$ Weyl transformations and a shift in $n \Gamma_{E_{8}}$. Thus

$$
\widetilde{Z}_{n}(\omega)=\sum_{a=1}^{F(n)} \widetilde{Z}_{n}^{a}(\omega),
$$

where

$$
\widetilde{Z}_{n}^{a}(\omega)=\sum_{d_{C}=n, \lambda_{C} \in \Delta^{a}} \mathcal{N}(C) e^{2 \pi i(C \cdot \omega)} .
$$

Since $\mathcal{N}(C)=\mathcal{N}(w(C))$, where $w \in W\left(E_{8}\right)$, therefore,

$$
\widetilde{Z}_{n}^{a}(\omega)=\sum_{d_{C}=n, \lambda_{C} \in \Delta^{a}} \mathcal{N}(C) \sum_{w \in W\left(E_{8}\right)} e^{2 \pi i(w(C) \cdot \omega)}
$$


The curves of degree $n$ are given by

$$
C=\sum_{a=1}^{8} \lambda_{a} \omega^{a}+n B+\left\{\frac{2 g-2+n+n^{2}+\lambda^{2}}{2 n}\right\} F,
$$

where $g=1+\frac{C^{2}-n}{2}$ and therefore,

$\widetilde{Z}_{n}^{a}(\omega)=e^{2 \pi i n \phi} \sum_{g, \lambda \in \Delta^{a}} \mathcal{N}_{n}^{a}(g) e^{2 \pi i \tau\left(\frac{2 g-2+n+n^{2}}{2 n}\right)} \sum_{w \in W\left(E_{8}\right)} e^{2 \pi i\left(\frac{\tau}{2 n} \lambda^{2}-(w(\lambda), m)\right)}$.

In the above expression $\mathcal{N}_{n}^{a}(g)$ is a function of degree $n$, genus $g$ and the dominant weight $\lambda^{a}$ since all vectors in $\Delta^{a}$ are Weyl equivalent to $\lambda^{a}$. Thus

$$
\begin{aligned}
\widetilde{Z}_{n}^{a}(\omega) & =q^{n / 2} e^{2 \pi i n \phi} \sum_{g} \mathcal{N}_{n}^{a}(g) q^{\frac{2 g-2+n}{2 n}} \sum_{\lambda \in \Delta^{a}} \sum_{w \in W\left(E_{8}\right)} q^{\frac{\lambda^{2}}{2 n}} e^{-2 \pi i(w(\lambda), m)}(22) \\
& =q^{n / 2} e^{2 \pi i n \phi} \widehat{Z}_{n}^{a}(\tau) P_{n, a}(\vec{m}, \tau)
\end{aligned}
$$

Where

$$
\begin{aligned}
\widehat{Z}_{n}^{a}(\tau) & =\sum_{g} \mathcal{N}_{n}^{a}(g) q^{\frac{2 g-2+n}{2 n}} \\
P_{n, a}(\vec{m}, \tau) & =\sum_{\lambda \in \Delta^{a}} \sum_{w \in W\left(E_{8}\right)} q^{\frac{\lambda^{2}}{2 n}} e^{-2 \pi i(w(\lambda), m)} .
\end{aligned}
$$

The functions $\widehat{Z}_{n}^{a}(\tau)$ can be determined from the genus zero topological string amplitude, $F_{0}(\omega)$. $F_{0}(\omega)$ can be calculated using local mirror symmetry as in $[12]$.

\subsection{E-string partition functions}

The E-string partition functions capture the BPS states of M5-branes wrapped on $\frac{1}{2} \mathrm{~K} 3 \times S^{1}$ with some momentum in the $S^{1}$ direction[12, 28, 29, 30]. The theory on $\frac{1}{2} \mathrm{~K} 3$ is twisted $\mathcal{N}=4$ Super Yang-Mills. Of the three possible twists of the $\mathcal{N}=4$ Yang-Mills the one that arises here was studied in [11]. It was shown in [11] that the partition function of this twisted theory is the generating function of the Euler characteristic of the instanton moduli spaces.

To relate the E-string partition function to the partition function of the $\mathcal{N}=4$ twisted theory consider a bundle $V$ of rank $r$ with $\Sigma$ as the 2-cycle dual to the first Chern class and instanton number $l$,

$$
\operatorname{ch}_{0}(V)=r, c_{1}(V)=\Sigma, \operatorname{ch}_{2}(V)=k:=\frac{1}{2} \Sigma \cdot \Sigma-l .
$$


Let $\mathcal{M}(r, \Sigma, l)$ be the moduli space of the bundle with above Chern classes and $e_{\Sigma}^{r}(l)$ be the Euler characteristic of the moduli space. We define the generating function for the Euler characteristic as [11]

$$
Z_{\Sigma}^{r}(\tau):=\sum_{l} e_{\Sigma}^{r}(l) q^{-\langle V, V\rangle / 2 r}=q^{-\frac{r}{2}} \sum_{l} e_{\Sigma}^{r}(l) q^{\Delta(l)}, \quad q=e^{2 \pi i \tau} .
$$

Where $\langle V, V\rangle=r^{2}-\Sigma \cdot \Sigma+2 r k$ and $\Delta(l)=l-\frac{r-1}{2 r} \Sigma \cdot \Sigma=-\frac{\langle V, V\rangle}{2 r}+\frac{r}{2}$. Define,

$$
\mathcal{Z}_{r, \vec{\lambda}}(\tau):=\frac{1}{r} \sum_{s=0}^{r-1} Z_{(\vec{\lambda}, 0, s)}^{r}(\tau),
$$

then $\mathcal{Z}_{r, \vec{\lambda}}(\tau)$ are the E-string partition functions. Note that the E-string partition function are given by generating function of instanton moduli space with first Chern class of degree zero. This is also the moduli-space of a configuration of D4-branes, D2-branes and D0-branes where the number of D4-branes is equal to the rank of the bundle, the D2-brane is wrapped on the 2-cycle dual to the first Chern class and the number of D0-branes is equal to the instanton number. However, since $\mathcal{B}_{9}$ is an elliptically fibered surface therefore T-duality on both fibers maps a wrapped D4-brane to a D2brane wrapped on the base $B$, a D0-brane becomes a D2-brane wrapping the fiber $F$ and a D2-brane wrapped on a curve $n B+k F$ maps to $n \mathrm{D} 4$-branes wrapped on $\mathcal{B}_{9}$ with $k$ D0-branes. Thus if the degree of the first Chern class (the cycle on which the D2-brane is wrapped) is zero the entire configuration of D4/D2/D0-branes can be mapped to a curve. Thus in this case the twisted Yang-Mills partition function can be related to curve counting function. In [12] this fact was used to relate the E-string partition functions, which are given by generating function of instanton moduli spaces with degree of the first Chern class zero, to the genus zero topological string amplitude as follow,

$$
(-1)^{r-1} \mathcal{Z}_{r, \lambda^{a}}(\tau)=\frac{q^{-n / 2}}{n^{3}} \delta_{\lambda^{a}, 0}+\widehat{Z}_{r}^{a}(\tau) .
$$

The first term which is only present for $\lambda^{a}=0$ is due to the fact that $n B$ is not a holomorphic curve for $n>1$ (it does not satisfy the adjunction formula) and therefore the corresponding term is not present in $\widehat{Z}_{n}^{0}(\tau)$ but genus zero topological string amplitude does contain such a term due to contributions coming from multiple cover of $B$.

The transformation property of $\mathcal{Z}_{r, \lambda}(\tau)$ under S-duality can be easily determined from that of $Z_{\Sigma}^{r}(\tau)$ [11],

$$
Z_{\Sigma}^{r}\left(-\frac{1}{\tau}\right)=(-1)^{r} r^{-5} \tau^{-6} \sum_{\Sigma^{\prime} \in H_{2}\left(\mathcal{B}_{9}, \mathbf{Z}_{r}\right)} e^{\frac{2 \pi i}{r} \Sigma \cdot \Sigma^{\prime}} Z_{\Sigma^{\prime}}^{r}(\tau) .
$$


This implies that

$$
\begin{aligned}
Z_{(\lambda, 0, s)}^{r}\left(-\frac{1}{\tau}\right)= & (-1)^{r} r^{-5} \tau^{-6} \sum_{\substack{\left(\mu, d, s^{\prime}\right) \\
\epsilon H_{2}\left(\mathcal{B}_{9}, \mathbf{Z}_{r}\right)}} e^{\frac{2 \pi i}{r}(-\lambda \cdot \mu+s d)} Z_{\left(\mu, d, s^{\prime}\right)}^{r}(\tau), \quad(29) \\
= & (-1)^{r} r^{-5} \tau^{-6} \sum_{\mu \in \Gamma_{8} / r \Gamma_{8}} \sum_{d, s^{\prime}=0}^{r-1} e^{\frac{2 \pi i}{r}(-\lambda \cdot \mu+s d)} Z_{\left(\mu, d, s^{\prime}\right)}^{r}(\tau), \\
= & (-1)^{r} r^{-5} \tau^{-6} \sum_{\mu \in \Gamma_{8} / r \Gamma_{8}} e^{-\frac{2 \pi i}{r} \lambda \cdot \mu}\left\{\sum_{s^{\prime}=0}^{r-1} Z_{\left(\mu, 0, s^{\prime}\right)}^{r}(\tau)+\right. \\
& \left.\sum_{\substack{d=1 \\
s^{\prime}=0}}^{r-1} e^{\frac{2 \pi i}{r} s d} Z_{\left(\mu, d, s^{\prime}\right)}^{r}(\tau)\right\} \\
= & (-1)^{r} r^{-5} \tau^{-6} \sum_{\mu \in \Gamma_{8} / r \Gamma_{8}} e^{-\frac{2 \pi i}{r} \lambda \cdot \mu}\left\{r \mathcal{Z}_{r, \mu}(\tau)+\right. \\
& \left.\sum_{\substack{d=1 \\
s^{\prime}=0}}^{r-1} e^{\frac{2 \pi i}{r} s d} Z_{\left(\mu, d, s^{\prime}\right)}^{r}(\tau)\right\} .
\end{aligned}
$$

Summing over $s$ on both sides of the equation and noting that the second term in the right hand side gives no contribution we find

$$
\mathcal{Z}_{r, \lambda}\left(-\frac{1}{\tau}\right)=(-1)^{r} r^{-4} \tau^{-6} \sum_{\mu \in \Gamma_{8} / r \Gamma_{8}} e^{-\frac{2 \pi i}{r} \lambda \cdot \mu} \mathcal{Z}_{r, \mu}(\tau)
$$

This implies that

$$
r^{4} \tau^{6} \mathcal{Z}_{r, 0}\left(-\frac{1}{\tau}\right)-\mathcal{Z}_{r, 0}(\tau)=\sum_{a=1}^{F(r)} C_{a} \mathcal{Z}_{r, \lambda^{a}}(\tau)
$$

Where $C_{a}$ is the number of elements in $\Delta^{a}$ and $\sum_{a=1}^{F(r)} C_{a}=r^{8}$. Also in writing the left hand side of eq(31) we have used eq(27) and the fact that multiplicity of the curve only depends on the degree, genus and the class of the weight vector. In [12] partition functions were calculated for $r=1,2,3,4$ by solving the holomorphic anomaly equation.

Rank one and two: Here we list the functions $\mathcal{Z}_{r, \lambda^{a}}$ for $r=1$ and $r=2$ 
$[12]$

$$
\begin{aligned}
\mathcal{Z}_{1,0}(\tau) & =\eta^{-12}(\tau) \\
\mathcal{Z}_{2,0}(\tau) & =-\frac{1}{24 \eta^{24}}\left(E_{2} P_{0}(\tau)+\left(\theta_{3}^{4} \theta_{4}^{4}-\frac{1}{8} \theta_{2}^{4}\right)\left(\theta_{3}^{4}+\theta_{4}^{4}\right)\right) \\
\mathcal{Z}_{2, \lambda_{\text {even }}} & =-\frac{1}{24 \eta^{24}}\left(\frac{1}{135} E_{2} P_{\text {even }}(\tau)-\frac{1}{8} \theta_{3}^{4}\left(\theta_{3}^{4}+\theta_{4}^{4}\right)\right) \\
\mathcal{Z}_{2, \lambda_{\text {odd }}} & =-\frac{1}{24 \eta^{24}}\left(\frac{1}{120} E_{2} P_{\text {odd }}(\tau)-\frac{1}{8} \theta_{2}^{4} E_{4}\right)
\end{aligned}
$$

There are three dominant weights of $E_{8}$ at level $2, \lambda=0, \lambda_{\text {even }}$ and $\lambda_{\text {odd }}$. Where $\lambda_{\text {even }}^{2}=4, \lambda_{\text {odd }}^{2}=2$ and $C_{\text {even }}=135, C_{\text {odd }}=120$. Definition of various function appearing in the equation above are given in appendix $\mathrm{C}$.

For $r=2$ it is possible to write $\mathcal{Z}_{2, \lambda_{\text {even }}}$ and $\mathcal{Z}_{2, \lambda_{\text {odd }}}$ in terms of $\mathcal{Z}_{2,0}(\tau)$. Define $F(\tau):=2^{4} \tau^{6} \mathcal{Z}_{2,0}\left(-\frac{1}{\tau}\right)-\mathcal{Z}_{2,0}(\tau)$ then from eq(31) it follows that

$$
\begin{aligned}
\sum_{a=1,2} C_{a} \mathcal{Z}_{2, \lambda^{a}}(\tau)= & F(\tau):=-\frac{1}{24 \eta^{24}(\tau)}\left\{\widehat{E}_{2}(\tau)\left(P_{0}\left(\frac{\tau}{4}\right)-P_{0}(\tau)\right)-\right. \\
& \left.2^{4}\left(\theta_{3}^{4} \theta_{2}^{4}-\frac{1}{8} \theta_{4}^{8}\right)\left(\theta_{3}^{4}+\theta_{2}^{4}\right)-\left(\theta_{3}^{4} \theta_{4}^{4}-\frac{1}{8} \theta_{2}^{8}\right)\left(\theta_{3}^{4}+\theta_{4}^{4}\right)\right\}
\end{aligned}
$$

Then from eq $(23)$ it follows that $\mathcal{Z}_{2, \lambda_{\text {odd }}}(\tau)$ has an expansion in integer plus half powers of $q$ whereas $\mathcal{Z}_{2, \lambda_{\text {even }}}(\tau)$ has an expansion in integer powers of $q$. Therefore,

$$
\begin{aligned}
\mathcal{Z}_{2, \lambda_{\text {odd }}}(\tau)= & -\frac{1}{2}(F(\tau)-F(\tau+1)) \\
= & \frac{1}{48 C_{\text {odd }} \eta^{24}}\left\{E_{2}(\tau)\left(E_{4}\left(\frac{\tau+1}{2}\right)-E_{4}\left(\frac{\tau}{2}\right)\right)+\right. \\
& \left.2^{4}\left(\theta_{4}^{4} \theta_{2}^{4}+\frac{1}{8} \theta_{3}^{8}\right)\left(\theta_{4}^{4}-\theta_{2}^{4}\right)+2^{4}\left(\theta_{3}^{4} \theta_{2}^{4}-\frac{1}{8} \theta_{4}^{8}\right)\left(\theta_{3}^{4}+\theta_{2}^{4}\right)\right\} \\
\mathcal{Z}_{2, \lambda_{\text {even }}}(\tau)= & -\frac{1}{2}(F(\tau)+F(\tau+1)) \\
= & \frac{1}{48 C_{\text {even }} \eta^{24}}\left\{E_{2}(\tau)\left(E_{4}\left(\frac{\tau+1}{2}\right)+E_{4}\left(\frac{\tau}{2}\right)-2 E_{4}(\tau)\right)+\right. \\
& 2^{4}\left(\theta_{4}^{4} \theta_{2}^{4}+\frac{1}{8} \theta_{3}^{8}\right)\left(\theta_{4}^{4}-\theta_{2}^{4}\right)-2^{4}\left(\theta_{3}^{4} \theta_{2}^{4}-\frac{1}{8} \theta_{4}^{8}\right)\left(\theta_{3}^{4}+\theta_{2}^{4}\right)- \\
& \left.2\left(\theta_{3}^{4} \theta_{4}^{4}-\frac{1}{8} \theta_{2}^{8}\right)\left(\theta_{3}^{4}+\theta_{4}^{4}\right)\right\}
\end{aligned}
$$

Note that both $\mathcal{Z}_{2, \text { even }}$ and $\mathcal{Z}_{2, \text { odd }}$ have integer expansion whereas $\mathcal{Z}_{2,0}$ does not. The non-integer expansion of $\mathcal{Z}_{2,0}$ is due to the fact that it contains contribution from multiple covers with weight $\frac{1}{n^{3}}$ whereas $\mathcal{Z}_{2, \text { odd }}$ and $\mathcal{Z}_{2, \text { even }}$ do not have any such multiple cover contribution and are sum over curves which are not multiples of some other curve. 


\section{Generalized Weyl transformations}

We saw in the last section that the affine Weyl transformations relating different classes in $H_{2}\left(\mathcal{B}_{9}\right)$ lead to an important organizing principle. The partition functions are organized in terms of level $n$ dominant weights with curves of same degree, genus and class of the dominant weight having the same multiplicity. In this section we show that there are other transformations which act not only on the second homology but on $H_{0} \oplus H_{2} \oplus H_{4}$. We call these transformation generalized Weyl transformations since they might be related to the Weyl transformations of the double loop algebra $\widehat{E}_{9}$ studied in $[33,34]$.

These transformations can be understood as the mirror of the Picard-Lefshetz transformations of the 3 -cycles in the mirror Calabi-Yau. Since $\mathcal{B}_{9}$ is elliptically fibered we can perform $S L(2, \mathbb{Z})$ transformations on the fiber. It was shown in [32] that the $S$-transformation acts like the Fourier-Mukai transformation on the Chern characters of a bundle and the $T$-transformation acts by tensoring the bundle with a line bundle $\mathcal{O}(B)$. Consider a bundle $V$ with Chern classes given by

$$
\operatorname{ch}_{0}(V)=r, \operatorname{ch}_{1}(V)=\Sigma=\{\vec{\lambda}, d, s\}, \operatorname{ch}_{2}(V)=k .
$$

Then the $S$ and the $T$ transformation maps these charges to [32]

$$
\begin{gathered}
S:(r,\{\lambda, d, s\}, k) \rightarrow\left(-d,\left\{w(\lambda), r,-k+\frac{1}{2} d\right\}, s-\frac{1}{2} r\right), \\
T:(r,\{\lambda, d, s\}, k) \rightarrow\left(r,\{w(\lambda), d+r, s\}, k+s-d-\frac{1}{2} r\right) .
\end{gathered}
$$

It is easy to check that the product $\langle V, V\rangle$ is invariant under both these transformations. The $S L(2, \mathbb{Z})$ action on the rank $r$ and the degree of the first Chern class $d$ is given by

$$
\begin{aligned}
& S:\left(\begin{array}{l}
d \\
r
\end{array}\right) \mapsto\left(\begin{array}{c}
r \\
-d
\end{array}\right), \\
& T: \quad\left(\begin{array}{l}
d \\
r
\end{array}\right) \mapsto\left(\begin{array}{c}
d+r \\
r
\end{array}\right) .
\end{aligned}
$$

The $S L(2, \mathbb{Z})$ transformation $\mathbf{- 1}$ changes the signs of all the charges. Thus it follows that any bundle (D4/D2/D0-brane configuration) can be mapped to a bundle with $r=0$ i.e., to a curve.

The $E_{8}$ algebra in $H_{2}\left(\mathcal{B}_{9}\right)$ is enhanced by the presence of $F$ (the imaginary root) to affine $E_{8}$ since $F^{2}=0$. Instead of enhancing the algebra using 
$F$ we can also use the charge vector $Q=(0,0,-1)$ (a 0 -cycle) to enhance the algebra since $\langle Q, Q\rangle=0$. Weyl transformations in roots involving $Q$ give more non-trivial action on the charges compared with the usual Weyl transformations as discussed in Appendix C.

$$
\begin{aligned}
G_{ \pm}:(0,\{\lambda, d, s\}, k) & \mapsto(0,\{\lambda, d, s\}, k \pm(\lambda, d)) \\
w:(0,\{\lambda, d, s\}, k) & \mapsto(0,\{w(\lambda), d, s\}, k), w \in W\left(E_{8}\right) .
\end{aligned}
$$

where $(\lambda, d)=d$ when $\lambda=0$, and $(\lambda, d)=\operatorname{gcd}(m, d)$ where $m$ is the largest integer for which $\lambda \in m \Gamma_{E_{8}}$. Let us consider an bundle $V_{1}$ such that degree of the first Chern class of $V_{1}$ is zero. Then using (35), (38), (35), and the $\mathbf{- 1}$ transformation successively we find

$$
\begin{aligned}
\operatorname{ch}\left(V_{1}\right)=(r,\{\vec{\lambda}, 0, s\}, k) & \mapsto\left(0,\left\{w(\vec{\lambda}), r, l+\frac{\lambda^{2}}{2}\right\}, s-\frac{r}{2}\right) \\
& \mapsto\left(0,\left\{\vec{\lambda}, r, l+\frac{\lambda^{2}}{2}\right\}, s-\frac{r}{2}+(r, \vec{\lambda})\right) \\
& \mapsto(r,\{\vec{\lambda}, 0, s+(r, \vec{\lambda})\}, k) .
\end{aligned}
$$

This implies that $e_{(\vec{\lambda}, 0, s)}^{r}(l)=e_{(\vec{\lambda}, 0, s+(r, \vec{\lambda}))}^{r}(l)$ and therefore

$$
Z_{(\vec{\lambda}, 0, s)}^{r}(\tau)=Z_{(\vec{\lambda}, 0, s+(r, \vec{\lambda}))}^{r}(\tau) .
$$

For the rank two case it follows that

$$
\begin{aligned}
Z_{\left(\lambda_{\text {even }}, 0,0\right)}^{2}(\tau) & =Z_{\left(\lambda_{\text {even }}, 0,1\right)}^{2}(\tau)=\mathcal{Z}_{2, \lambda_{\text {even }}}(\tau), \\
Z_{\left(\lambda_{\text {odd }}, 0,0\right)}^{2}(\tau) & =Z_{\left(\lambda_{\text {odd }}, 0,1\right)}^{2}(\tau)=\mathcal{Z}_{2, \lambda_{\text {odd }}}(\tau) .
\end{aligned}
$$

This result (rank two case) was also given in [35] where $\mathcal{Z}_{2,0}(\tau), \mathcal{Z}_{2, \lambda_{\text {even }}}(\tau)$ and $\mathcal{Z}_{2, \lambda_{\text {odd }}}(\tau)$ were also obtained by a rigorous mathematical analysis.

\section{Partition functions}

In this section we use the transformation discussed in the previous section to obtain generating functions of the Euler characteristic of the instanton moduli spaces for various ranks.

rank one: This case has been discussed in several places $[11,12]$ but we will consider it to illustrate the method. Consider a rank one bundle such that

$$
\operatorname{ch}_{0}(V)=1, \operatorname{ch}_{1}(V):=\Sigma=\{\vec{\lambda}, d, s\}, \operatorname{ch}_{2}(V)=k=\frac{1}{2} \Sigma \cdot \Sigma-l .
$$


Since zero is the only dominant vector at level one, by an affine $E_{8}$ Weyl transformation we can set the $\vec{\lambda}$ in the equation above to zero. Also since the degree of the first Chern class and the rank is relatively prime therefore by an $S L(2, \mathbb{Z})$ transformation we can map the above bundle to a bundle with rank zero. Thus the above bundle is mapped to $V_{1}$ such that

$$
\operatorname{ch}_{0}\left(V_{1}\right)=0, \operatorname{ch}_{1}\left(V_{1}\right)=\{0,1, l\}, \operatorname{ch}_{2}\left(V_{1}\right)=s-\frac{1}{2} .
$$

Since the transformation $G_{-}$allows us to change $s$ in the equation above by -1 therefore by repeated application of $G_{-}$we get

$$
\operatorname{ch}\left(V_{2}\right)=\left(0,\{0,1, l\},-\frac{1}{2}\right) .
$$

Now the $S$ transformation maps it back to a rank one bundle $V_{3}$ with

$$
\operatorname{ch}\left(V_{3}\right)=(1,\{0,0,0\},-l) .
$$

And therefore $e_{\Sigma}^{1}(l)=e_{(0,0,0)}^{1}(l)$.

To determine $e_{(0,0,0)}^{1}$ note that the bundle with these charge can be realized a bound states of a D4-brane and $l$ D0-branes. Thus the moduli space is give by the $l-t h$ symmetric product of $\mathcal{B}_{9}[11,12] .{ }^{4}$. And therefore the Euler characteristic is given by

$$
\sum_{l=0}^{\infty} \chi\left(\operatorname{Sym}^{l}\left(\mathcal{B}_{9}\right)\right) q^{l}=\prod_{n=1}^{\infty}\left(1-q^{n}\right)^{-12}=q^{1 / 2} \eta^{-12}(\tau)=\sum_{l=0}^{\infty} d(l) q^{l} .
$$

Thus $e_{(0,0,0)}^{1}(l)=d(l)$ and eq(25) implies that

$$
\begin{aligned}
Z_{\Sigma}^{1}(\tau) & =q^{-1 / 2} \sum_{l} e_{\Sigma}^{1}(l) q^{l} \\
& =q^{-1 / 2} q^{1 / 2} \eta^{-12}(\tau)=\eta^{-12}(\tau)
\end{aligned}
$$

rank $\mathbf{r}$, degree $d$ with $\operatorname{gcd}(d, r)=1$ : Now consider a bundle $V$ with rank $r$ and degree of the first Chern class $d$ such that $r$ and $d$ are relatively prime. Then since the $S L(2, \mathbf{Z})$ transformation acts on $r$ and $d$ we can map this bundle to a bundle with rank one. As discussed in the previous section, any bundle of rank one can be mapped to a bundle of rank 1 with zero first Chern class therefore,

$$
\operatorname{ch}(V)=(r, \Sigma=\{\lambda, d, s\}, k) \mapsto \operatorname{ch}\left(V_{1}\right)=\left(1,\{0,0,0\}, l_{1}\right) .
$$

\footnotetext{
${ }^{4}$ This is also the moduli space of degree one curve $B+l F$ if we also include the flat connections on this genus $l$ curve [37]
} 
Where $l_{1}$ can be determined by the fact that all transformation we have used preserve the product $\langle V, V\rangle=\left\langle V_{1}, V_{1}\right\rangle$. This gives $\left(k=\frac{1}{2} \Sigma \cdot \Sigma-l\right)$

$$
l_{1}=-\frac{\langle V, V\rangle}{2}+\frac{1}{2}=r l-\frac{1}{2}(r-1) \Sigma^{2}-\frac{1}{2}\left(r^{2}-1\right) .
$$

We then have $(d=F \cdot \Sigma$ and is relatively prime to $r)$,

$$
\begin{aligned}
Z_{\Sigma}^{r}(\tau) & =\sum_{l} e_{\Sigma}^{r}(l) q^{-\frac{\langle V, V\rangle}{2 r}} \\
& =q^{-\frac{1}{2 r}} \sum_{l_{1}=0}^{\infty} d\left(l_{1}\right) q^{\frac{l_{1}}{r}}
\end{aligned}
$$

Note that writing $l_{1}=r l+\mu$ we see that the partition function for fixed rank only depends on $\mu(\bmod \mathrm{r})$ and only $\Sigma^{2}$ enters in the determination of $\mu$. Using the identity

$$
\sum_{k=0}^{r-1} \omega_{r}^{(2 g-2 s) k}=r \delta_{g \equiv s(\bmod r)}, \quad \omega_{r}=e^{\frac{i \pi}{r}} .
$$

We get

$$
\begin{aligned}
Z_{\Sigma}^{r}(\tau) & =q^{-\frac{1}{2 r}+\frac{\mu}{r}} \sum_{r l+\mu \geq 0} d(r l+\mu) q^{l} \\
& =\frac{1}{r} \sum_{m=0}^{r-1} \omega_{r}^{(1-2 \mu) m} \eta^{-12}\left(\frac{\tau}{r}+\frac{m}{r}\right)
\end{aligned}
$$

Since $\mu=-\frac{1}{2}\left(r^{2}-1\right)-\frac{r-1}{2} \Sigma^{2}$ therefore

$$
Z_{\Sigma}^{r}(\tau)=\frac{1}{r} \sum_{m=0}^{r-1}(-1)^{r m} e^{i \pi m(r-1) \Sigma^{2} / r} \eta^{-12}\left(\frac{\tau}{r}+\frac{m}{r}\right)
$$

rank $\mathbf{r}$, degree $d=0$ : Consider a bundle of rank $r$ with degree of the first Chern class equal to zero. These were the configuration considered in [12]. Using the $S$-transformation we can map this bundle to a curve of degree $r$.

From eq(30) it follows that

$$
Z_{(\lambda, 0, s)}^{r}(\tau)=\mathcal{Z}_{r, \lambda}(\tau)+(-1)^{r} r^{-5} \tau^{6} \sum_{\mu \in \Gamma_{8} / r \Gamma_{8}} \sum_{\substack{d=1 \\ s^{\prime}=0}}^{r-1} e^{\frac{2 \pi i}{r}(-\lambda \cdot \mu+s d)} Z_{\left(\mu, d, s^{\prime}\right)}^{r}\left(-\frac{1}{\tau}\right) .
$$

Specializing to prime rank $r=p$ and using eq(53) one finds

$$
Z_{(\lambda, 0, s)}^{p}(\tau)=\mathcal{Z}_{p, \lambda}(\tau)-\frac{(-1)^{p}}{p^{11}}\left(\sum_{\mu \in \Gamma_{8} / r \Gamma_{8}} e^{-2 \pi i \frac{\lambda \cdot \mu}{p}}\right) \omega(p, s) \eta^{-12}(p \tau)
$$


where

$$
\omega(p, s)=\sum_{d=1}^{p-1} e^{2 i \pi s d / p}= \begin{cases}p-1, & \text { if } s=0 \\ -1, & \text { if } s \neq 0\end{cases}
$$

The sum in parenthesis in (55) can be evaluated explicitly to obtain,

$$
Z_{(\lambda, 0, s)}^{p}(\tau)=\mathcal{Z}_{p, \lambda}(\tau)-\frac{(-1)^{p} \delta_{\vec{\lambda}, p}}{p^{3}} \omega(p, s) \eta^{-12}(p \tau) .
$$

Here $\delta_{\vec{\lambda}, p}$ vanishes unless $\lambda \in p \Gamma_{E_{8}}$ in which case it takes the value one.

examples: For the rank two case this give

$$
\begin{aligned}
Z_{\Sigma}^{2}(\tau) & =\frac{1}{2}\left\{\eta^{-12}\left(\frac{\tau}{2}\right)+i^{\Sigma^{2}} \eta^{-12}\left(\frac{\tau}{2}+\frac{1}{2}\right)\right\}, \quad \mathrm{d}_{\Sigma} \in 2 \mathbb{Z}+1, \\
Z_{(\vec{\lambda}, 0, s)}^{2}(\tau) & =\mathcal{Z}_{2, \vec{\lambda}}(\tau)+(-1)^{s+1} \frac{\delta_{\vec{\lambda}, 2}}{8} \eta^{-12}(2 \tau) .
\end{aligned}
$$

This result for the rank two case was also obtained in [35]. A conjecture about the $U(r)$ partition function for $r$ an odd prime was made in [23]. For rank three we have $\left(\mathrm{d}_{\Sigma}=\Sigma \cdot F \neq 0(\bmod 3)\right)$

$$
\begin{aligned}
Z_{\Sigma}^{3}(\tau) & =\frac{1}{3}\left\{\eta^{-12}\left(\frac{\tau}{3}\right)-e^{\frac{2 i \pi}{3} \Sigma^{2}} \eta^{-12}\left(\frac{\tau}{3}+\frac{1}{3}\right)+e^{\frac{4 i \pi}{3} \Sigma^{2}} \eta^{-12}\left(\frac{\tau}{3}+\frac{2}{3}\right)\right\},, \\
Z_{(\lambda, 0, s)}^{3}(\tau) & =\mathcal{Z}_{3, \lambda}(\tau)+\frac{\delta_{\vec{\lambda}, 3}}{27} \omega(3, s) \eta^{-12}(3 \tau), \\
\text { where } \omega(3,0) & =2 \text { and } \omega(3,1)=\omega(3,2)=-1 .
\end{aligned}
$$

\subsection{A recursive scheme}

For prime rank $p$ the complete explicit solution can be obtained in terms of $\eta^{-12}(\tau)$ and the E-string partition functions as shown in the previous subsection. For non prime rank $r$ there are three cases. When $\operatorname{gcd}(r, \mathrm{~d})=1$ we use (53). When the $\operatorname{gcd}(r, \mathrm{~d})=f>1$, we can map such bundles to rank $f$ (which is less than $r$ ) bundles. So these can be written in terms of lower rank partition functions. When the degree $d$ is zero we simply use (54) which expresses the partition function in terms of an E-string partition function and (by the above argument) partition functions of lower rank.

Consider a rank $r$ bundle $V$ of first Chern class $\Sigma$ such that $\operatorname{gcd}\left(r, \mathrm{~d}_{\Sigma}\right)=f$. Then using (35) and (36) we can map $V$ to a rank $f$ bundle

$$
\left(r,\left\{\lambda, \mathrm{d}_{\Sigma}, s\right\}, k\right) \mapsto\left(f,\{\lambda, 0, s(l)\},-\frac{\lambda^{2}}{2}-l^{\prime}\right),
$$


where

$$
l^{\prime}=r_{0} l+\mu, \quad r_{0}=\frac{r}{f}, \quad \mu=-\frac{r-1}{2 f} \Sigma^{2}-\frac{r^{2}}{2 f}+\frac{f}{2}-\frac{f-1}{2 f} \lambda^{2} .
$$

which implies that $e_{\Sigma}^{r}(l)=e_{(\lambda, 0, s(l))}^{f}\left(l^{\prime}\right)$.

$$
\begin{aligned}
Z_{\Sigma}^{r}(\tau) & =q^{-\frac{r}{2}-\frac{r-1}{2 r} \Sigma^{2}} \sum_{l} e_{\Sigma}^{r}(l) q^{l} \\
& =q^{-\frac{r}{2}-\frac{r-1}{2 r} \Sigma^{2}} \sum_{l} e_{(\lambda, 0, s(l)}^{r}\left(r_{0} l+\mu\right) q^{l} \\
& =q^{\frac{\mu}{r_{0}}} q^{-\frac{f}{2 r_{0}}-\frac{f-1}{2 f r_{0}} \lambda^{2}} \sum_{l} e_{(\lambda, 0, s(l))}^{f}\left(r_{0} l+\mu\right) q^{l}
\end{aligned}
$$

Let $h=(\vec{\lambda}, f)$ and define

$$
s^{\prime}(l) \equiv s(l)(\bmod h)
$$

Since $s(l)=a l+b$ with integers $a, b$, the argument of $s^{\prime}$ can be restricted to the integers $\bmod h$. Then we get

$$
\begin{aligned}
Z_{\Sigma}^{r}(\tau) & =q^{\frac{\mu}{r_{0}}} q^{-\frac{f}{2 r_{0}}-\frac{f-1}{2 f r_{0}} \lambda^{2}} \sum_{n=0}^{h-1} \sum_{l=n(\bmod h)} e_{\left(\lambda, 0, s^{\prime}(n)\right)}^{f}\left(r_{0} l+\mu\right) q^{l} \\
& =q^{\frac{\mu}{r_{0}}} q^{-\frac{f}{2 r_{0}}-\frac{f-1}{2 f r_{0}} \lambda^{2}} \sum_{n=0}^{h-1} \sum_{l} e_{\left(\lambda, 0, s^{\prime}(n)\right)}^{f}\left(r_{0} h l+r_{0} n+\mu\right) q^{h l+n} .
\end{aligned}
$$

It is not difficult to show that

$$
\begin{gathered}
q^{\frac{\mu}{r_{0}}-\frac{f}{2 r_{0}}-\frac{f-1}{2 f r_{0}} \lambda^{2}} \sum_{l} e_{\left(\lambda, 0, s^{\prime}(n)\right)}^{f}\left(r_{0} h l+r_{0} n+\mu\right) q^{h l+n}= \\
\frac{1}{r_{0} h} \sum_{m=0}^{r_{0} h-1} e^{\frac{2 \pi i m}{h}\left(\frac{r-1}{2 r} \Sigma^{2}+\frac{r}{2}-n\right)} Z_{\left(\lambda, 0, s^{\prime}(n)\right)}^{f}\left(\frac{\tau}{r_{0}}+\frac{m}{r_{0} h}\right) .
\end{gathered}
$$

Thus we get

$$
Z_{\Sigma}^{r}(\tau)=\frac{1}{r_{0} h} \sum_{n=0}^{h-1} \sum_{m=0}^{r_{0} h-1} e^{\frac{\pi i m r}{h}} e^{\frac{i \pi m}{h}\left(\frac{r-1}{r}\right) \Sigma^{2}} e^{-\frac{2 \pi i m n}{h}} Z_{\left(\lambda, 0, s^{\prime}(n)\right)}^{f}\left(\frac{\tau}{r_{0}}+\frac{m}{r_{0} h}\right) .
$$

example: Consider the case of rank $r=4$, For $\mathrm{d}_{\Sigma} \equiv 1,3(\bmod 4)$ it follows from eq(53),

$$
Z_{\Sigma}^{4}(\tau)=\frac{1}{4}\left\{\eta^{-12}\left(\frac{\tau}{4}\right)+e^{\frac{3 i \pi}{4} \Sigma^{2}} \eta^{-12}\left(\frac{\tau}{4}+\frac{1}{4}\right)+e^{\frac{6 i \pi}{4} \Sigma^{2}} \eta^{-12}\left(\frac{\tau}{4}+\frac{2}{4}\right)+e^{\frac{9 i \pi}{4} \Sigma^{2}} \eta^{-12}\left(\frac{\tau}{4}+\frac{3}{4}\right)\right\},
$$


for $\mathrm{d}_{\Sigma} \equiv 2(\bmod 4)$ it follows from eq $(66)$,

$Z_{\Sigma}^{4}(\tau)=\frac{1}{2}\left\{\mathcal{Z}_{2, \lambda}\left(\frac{\tau}{2}\right)+e^{\frac{3 i \pi}{4} \Sigma^{2}} \mathcal{Z}_{2, \lambda}\left(\frac{\tau}{2}+\frac{1}{2}\right)\right\}-\frac{\delta_{\vec{\lambda}, 2}}{16}\left\{e^{\frac{3 i \pi}{4} \Sigma^{2}}-e^{\frac{9 i \pi}{8} \Sigma^{2}}\right\} \eta^{-12}\left(\tau+\frac{1}{2}\right)$,

for $\mathrm{d}_{\Sigma}=0$ we get using eq(54)

$Z_{(\lambda, 0, s)}^{4}(\tau)=\mathcal{Z}_{4, \lambda}(\tau)+\delta_{\vec{\lambda}, 2} \frac{(-1)^{s}}{2^{3}} \mathcal{Z}_{2, \lambda / 2}(2 \tau)-\delta_{\vec{\lambda}, 4} i^{s} \frac{\left(1+(-1)^{s}\right)}{4^{3}} \eta^{-12}(4 \tau)$

\section{Acknowledgment}

I would like to thank Jacques Distler, Tamás Hauer and Cumrun Vafa for many valuable discussions. I would specially like to thank Barton Zwiebach for many valuable discussions and collaboration in the early part of the project. This research is supported by the National Science Foundation under Grant No. 0071512.

\section{Appendix A}

Let $C$ be a class of degree $n$ satisfying the adjunction formula with genus $g$ then

$$
C=\sum_{i=1}^{8} \mu_{i} \omega^{i}+n B+\frac{1}{2 n}\left\{2 g-2+n+n^{2}+\vec{\mu}^{2}\right\} F
$$

Every degree one class can be represented by a holomorphic curve. This follows from the fact that $B+k F$ is holomorphic for $k \geq 0$ and this class is Weyl equivalent to $\sum_{i=1}^{8} \lambda_{i} \omega^{i}+B+s F$ for any $\lambda \in \Gamma_{E_{8}}$. The necessary and sufficient condition for a class to be represented by a holomorphic curve is that its intersection with exceptional curves be positive. Let $E$ be an exceptional curve with $E_{8}$ weight vector $\lambda$, then

$$
C \cdot E=\frac{n}{2}\left(\vec{\lambda}-\frac{\vec{\mu}}{n}\right)^{2}+\frac{2 g-2+n-n^{2}}{2 n} .
$$

$C$ has a holomorphic representative if this intersection number is nonnegative for all $\lambda \in \Gamma_{E_{8}}$.

$$
C \cdot E \geq \frac{n}{2} \operatorname{Min}_{\lambda \in \Gamma_{E_{8}}}\left(\lambda-\frac{\mu}{n}\right)^{2}+\frac{2 g-2+n-n^{2}}{2 n} .
$$


Define

$$
f_{n}(\mu)=\operatorname{Min}_{\lambda \in \Gamma_{E_{8}}}\left(\lambda-\frac{\mu}{n}\right)^{2} .
$$

Note that if $\mu=w\left(\mu^{\prime}\right)+n \nu$ then $f_{n}(\mu)=f_{n}\left(\mu^{\prime}\right)$ where $\nu \in \Gamma_{E_{8}}$ and $w \in$ $W\left(E_{8}\right)$. This implies that $f_{n}(\mu)=f_{n}\left(\mu^{a}\right)$, where $\mu$ belongs to the class $\mu^{a}$. 5

It follows that $g=g_{c}\left(n, \mu^{a}\right)+n m, m \in \mathbb{Z}$ and

$$
g_{c}\left(n, \mu^{a}\right):=1+\frac{1}{2}\left(n^{2}-n^{2} f_{n}\left(\mu^{a}\right)-n\right),
$$

where $g_{c}\left(n, \mu^{a}\right)$ is the genus of the degree $n$ curve with weight vector $\mu^{a}$. Thus we see that $C \cdot E$ is non-negative iff

$$
g \geq g_{c}\left(n, \mu^{a}\right) .
$$

If $C_{1}$ and $C_{2}$ are two curves satisfying the adjunction formula and eq(73) then $C_{1} \cdot C_{2} \geq 0$. To see this note that since $C_{1}$ and $C_{2}$ satisfy the adjunction formula therefore

$$
\begin{aligned}
& C_{1}=\sum_{i=1}^{8} \mu_{i} \omega_{i}+n_{1} B+\frac{2 g_{1}-2+n_{1}+n_{1}^{2}+\mu^{2}}{2 n_{1}} F \\
& C_{2}=\sum_{i=1}^{8} \nu_{i} \omega_{i}+n_{2} B+\frac{2 g_{2}-2+n_{2}+n_{2}^{2}+\nu^{2}}{2 n_{2}} F .
\end{aligned}
$$

The mutual intersection number is

$$
\begin{aligned}
C_{1} \cdot C_{2}= & -\vec{\mu} \cdot \vec{\nu}-n_{1} n_{2}+\frac{n_{1}}{2 n_{2}}\left\{2 g_{2}-2+n_{2}+n_{2}^{2}+\nu^{2}\right\}+ \\
& \frac{n_{2}}{2 n_{1}}\left\{2 g_{1}-2+n_{1}+n_{1}^{2}+\mu^{2}\right\} .
\end{aligned}
$$

Since $C_{1}$ and $C_{2}$ obey eq(73) therefore,

$$
g_{1} \geq g_{c}\left(n_{1}, \lambda^{a}\right) \text { and } g_{2} \geq g_{c}\left(n_{2}, \lambda^{b}\right)
$$

where $\lambda^{a}$ and $\lambda^{b}$ are the level $n_{1}$ and level $n_{2}$ dominant weights corresponding to the weight vectors $\mu$ and $\nu$ respectively. From these two conditions we get

$$
C_{1} \cdot C_{2} \geq n_{1} n_{2}\left\{1-\frac{1}{2}\left(f_{\lambda^{a}}\left(n_{1}\right)+f_{\lambda^{b}}\left(n_{2}\right)\right)+\frac{1}{2}\left(\frac{\nu}{n_{2}}-\frac{\mu}{n_{1}}\right)^{2}\right\}
$$

\footnotetext{
${ }^{5}$ Dominant weights $\lambda$ at level $n$ are given by $\sum_{i=1}^{8} a_{i} \lambda_{i} \leq n$, where $a_{i}$ are the Dynkin labels of $E_{8}$ and $\lambda_{i} \geq 0$. If $F(n)$ is the number of dominant weights at level $n$ then generating function is given by $\sum_{n=1}^{\infty} F(n) q^{n}=(1-q)^{-1} \prod_{i=1}^{8}\left(1-q^{a_{i}}\right)^{-1}$.
} 
Since $f_{\lambda^{a}}(n)=\min _{\lambda \in \Gamma_{8}}\left(\lambda-\frac{\lambda^{a}}{n}\right)$ therefore $f_{\lambda^{a}}(n) \leq\left(\frac{\lambda^{a}}{n}\right)^{2}$. The dominant weights satisfy the condition

$$
\sum_{i=1}^{8} a_{i} \lambda_{i}=2\left(\lambda_{1}+\lambda_{7}\right)+3\left(\lambda_{2}+\lambda_{8}\right)+4\left(\lambda_{3}+\lambda_{6}\right)+5 \lambda_{4}+6 \lambda_{5} \leq n .
$$

if we define the matrix $B$ as follows

$$
\begin{aligned}
B_{i j} & =2 a_{i} a_{j}, i \neq j, \\
& =a_{i}^{2}, i=j .
\end{aligned}
$$

Then we see that $B_{i j} \lambda_{i} \lambda_{j} \leq n^{2}$ and since $B_{i j}-\left(A_{E_{8}}^{-1}\right)_{i j} \geq 0$ therefore it follows that $\lambda^{2} \leq n^{2}$ if $\lambda$ is a level $n$ dominant weight. Thus we get $f_{n}(\mu) \leq 1$. Which therefore implies that,

$$
\begin{aligned}
C_{1} \cdot C_{2} & \geq n_{1} n_{2}\left\{1-\frac{1}{2}\left(f_{\lambda^{a}}\left(n_{1}\right)+f_{\lambda^{b}}\left(n_{2}\right)\right)+\frac{1}{2}\left(\frac{\nu}{n_{2}}-\frac{\mu}{n_{1}}\right)^{2}\right\}, \\
& \geq n_{1} n_{2}\left\{1-\frac{1}{2}\left(f_{\lambda^{a}}\left(n_{1}\right)+f_{\lambda^{b}}\left(n_{2}\right)\right)\right\} \geq 0 .
\end{aligned}
$$

From the fact that $\left(\lambda^{a}\right)^{2} \leq n^{2}$ it follows that $\lambda^{a}$ is the vector of least length in its class and therefore

$$
f_{n}(\lambda)=\frac{\left(\lambda^{a}\right)^{2}}{n^{2}}, \forall \lambda \in \lambda^{a}+n \Gamma_{E_{8}} .
$$

Then from $\mathrm{Eq}(72)$ it follows that

$$
g_{c}\left(n \lambda^{a}\right)=1+\frac{n(n-1)}{2}-\frac{\left(\lambda^{a}\right)^{2}}{2} .
$$

\section{Appendix B}

The Calabi-Yau space $X$ whose compact divisor is the rational elliptic surface, $\mathcal{B}_{9}$, is the total space of the canonical bundle over $\mathcal{B}_{9}$. The mirror manifold $Y$ is given by $[12,31,32]$

$$
\begin{aligned}
y^{2} & =x^{3}+f_{4}(z) x+g_{6}(z), \\
u v & =z-z_{*} .
\end{aligned}
$$

Where $z_{*}$ is a function of the Kähler moduli of $X$. The first equation defines an elliptic fibration with twelve singular fibers. Apart from the fact that the base space given by $z$ is non-compact (and therefore the total manifold is hyper Kähler) the geometry of the singular fibers is exactly the same as 
the compact $\frac{1}{2} \mathrm{~K} 3$. The twelve degenerate fibers can be chosen to have the following charges ${ }^{6}$,

$$
[1,0][1,0] \cdots[1,0][2,-1],[-1,-1],[-1,2] .
$$

It was shown in $[33,34]$ that this configuration is invariant under $S L(2, \mathbb{Z})$ transformations. The effect of this $S L(2, \mathbf{Z})$ transformation on the charges was determined in [32] and is given in $\operatorname{Eq}(36)$.

The transformations $G_{ \pm}$are more interesting and correspond to generalized Weyl transformation. Consider the lattice $H_{*}(X, \mathbf{Z})=H_{0}(X, \mathbb{Z}) \oplus$ $H_{2}(X, \mathbb{Z}) \oplus H_{4}(X, \mathbb{Z})$. A codimension one lattice in $H_{2}(X)$, orthogonal to the canonical class, is isomorphic to the root lattice of the affine $E_{8}$ algebra. One way of seeing this is that we have the ordinary $E_{8}$ algebra which is enhanced to affine $E_{8}$ by the canonical class , $F$, since $F^{2}=0$. Another way of obtaining the affine $E_{8}$ is to find another element of $H_{*}(X)$ which squares to zero. It is easy to see that only other such element is the generator of $H_{0}(X)$. Thus we have two different copies of affine $E_{8}$ root lattice in $H_{*}(X)$. Thus the most general root of $H_{*}(X)$ is given by (using the same notation as in section 3 )

$$
\widehat{\alpha}_{n, m}=(0,\{\alpha, 0, n\}, m), \alpha^{2}=2, n, m \in \mathbb{Z} .
$$

It is clear that $\left\langle\widehat{\alpha}_{n, m}, \widehat{\alpha}_{n, m}\right\rangle=-2$ and therefore $\langle\widehat{V}, \widehat{V}\rangle=\langle V, V\rangle$ where $\widehat{V}=V+\left\langle V, \widehat{\alpha}_{n, m}\right\rangle \widehat{\alpha}_{n, m}$. Two successive transformation using first the root $\widehat{\alpha}_{n, m}$ and then $\widehat{\alpha}_{n, 0}$ gives

$$
V:=(0,\{\lambda, d, s\}, k) \mapsto \widehat{V}=(0,\{\lambda, d, s\}, k+m(-\lambda \cdot \alpha+d n)\}
$$

Thus we see that if $\lambda \in h \Gamma_{E_{8}}$ then $k$ can be changes in units of $\operatorname{gcd}(h, d)$ by generalized Weyl transformations.

\section{Appendix $\mathrm{C}$}

$$
\begin{aligned}
\theta_{2}(\tau) & :=\sum_{n \in \mathbb{Z}} q^{\frac{\left(n-\frac{1}{2}\right)^{2}}{2}}, \theta_{3}(\tau):=\sum_{n \in \mathbb{Z}} q^{\frac{n^{2}}{2}}, \theta_{4}(\tau):=\sum_{n \in \mathbf{Z}}(-1)^{n} q^{\frac{n^{2}}{2}}(87) \\
\theta_{2}^{4}\left(-\frac{1}{\tau}\right) & =-\tau^{2} \theta_{4}^{4}(\tau), \theta_{3}^{4}\left(-\frac{1}{\tau}\right)=-\tau^{2} \theta_{3}^{4}(\tau), \theta_{4}^{4}\left(-\frac{1}{\tau}\right)=-\tau^{2} \theta_{2}^{4}(\tau) .
\end{aligned}
$$

\footnotetext{
${ }^{6}$ With respect to a particular choice of paths [33, 34].
} 


$$
\begin{gathered}
E_{2}(\tau)=1-24 \sum_{k=1}^{\infty} \sigma_{1}(k) q^{k} \\
E_{4}(\tau)=1+240 \sum_{k=1}^{\infty} \sigma_{3}(k) q^{k}, E_{4}\left(-\frac{1}{\tau}\right)=\tau^{4} E_{4}(\tau), \sigma_{s}(k):=\sum_{n \mid k} n^{s} \\
\left.P_{2,0}(\tau)=E_{4}(2 \tau), P_{2, \lambda^{1}}(\tau)=\frac{E_{4}\left(\frac{\tau}{2}\right)+E_{4}\left(\frac{\tau}{2}+\frac{1}{2}\right)}{2}-E_{4}(2 \tau), \quad 89\right) \\
P_{2, \lambda^{2}}(\tau)=\frac{E_{4}\left(\frac{\tau}{2}\right)-E_{4}\left(\frac{\tau}{2}+\frac{1}{2}\right)}{2}, \lambda^{1} \cdot \lambda^{1}=4, \lambda^{2} \cdot \lambda^{2}=2
\end{gathered}
$$

\section{References}

[1] P. S. Aspinwall, D. R. Morrison, "Topological Field Theory and Rational Curves", Commun. Math. Phys. 151 (1993) 245-262, hep-th/9110048.

[2] M. Bershadsky, S. Cecotti, H. Oguri, C. Vafa, "Kodaira-Spencer Theory of Gravity and Exact Results for Quantum String Amplitudes", Commun. Math. Phys. 165 (1994) 311-428, hep-th/9309140.

[3] S. Katz, A. Klemm, C. Vafa, "M-Theory, Topological Strings and Spinning Black Holes", Adv. Theor. Math. Phys. 3 (1999) 1445-1537, hep-th/9910181.

[4] M. Aganagic, C. Vafa, "Mirror Symmetry D-Branes and Counting Holomorphic Discs", hep-th/0012041;

M. Aganagic, A. Klemm, C. Vafa, "Disk Instantons, Mirror Symmetry and the Duality Web", Z. Naturforsch. A56 (2002) 1-28, hep-th/0105045.

[5] P. Mayr, "N=1 Mirror Symmetry and Open/Closed String Duality", hep-th/0108229;

W. Lerche, P.Mayr, "On N=1 Mirror Symmetry for Open Type II Strings", hep-th/0111113;

P.Mayr, "Summing up Open String Instantons and N=1 String Amplitudes", hep-th/0203237.

[6] S. Govindarajan, T. Jayaraman, T. Sarkar, "Disc Instantons in Linear Sigma Models", hep-th/0108234.

[7] A. Iqbal, A.-K. Kashani-Poor, "Discrete Symmetries of the Superpotential and Calculation of Disk Invariants", Adv. Theor. Math. Phys. 5 (2001), hep-th/0109214.

[8] J. Blum, "Calculation of Nonperturbative Terms in Open String Models", hep-th/0112039.

[9] B. Acharya, M. Aganagic, K. Hori, C. Vafa, "Orientifolds, Mirror Symmetry and Superpotentials", hep-th/0202208. 
[10] D. -E. Diaconescu, B. Florea, A. Grassi, "Geometric Transitions and Open String Instantons", hep-th/0205234.

[11] C. Vafa, E. Witten, "A Strong Coupling Test of S-Duality", Nucl. Phys. B431 (1994) 3-77, hep-th/9408074.

[12] J. A. Minahan, D. Nemeschansky, C. Vafa, N. P. Warner, "E-Strings and $N=4$ Topological Yang-Mills Theories", Nucl. Phys. B527 (1998) 581-623, hep-th/9802168.

[13] M. Jinzenji, T. Sasaki, “ $N=4$ Supersymmetric Yang-Mills Theory on Orbifold $T^{4} / \mathbf{Z}_{2}$ ", Mod. Phys. Lett. A16 (2001) 411-428, hep-th/0012242.

[14] M. Jinzenji, T. Sasaki, " $\mathcal{N}=4$ Supersymmetric Yang-Mills Theory on Orbifold $T^{4} / \mathbf{Z}_{2}$ : Higher Rank Case", JHEP 0112 (2001) 002, hep-th/0109159.

[15] M. Jinzenji, T. Sasaki, "An approach to $\mathcal{N}=4$ ADE Gauge Theory on K3", hep-th/0203179.

[16] R. Dijkgraaf, G. Moore, "Balanced Topological Field Theories", Commun. Math. Phys. 185 (1997) 411-440, hep-th/9608169.

[17] R. Dijkgraaf, J.-S. Park, B. Schroers, "N=4 Supersymmetric Yang-Mills Theory on a Kaehler Surface", hep-th/9801066.

[18] R. Dijkgraaf, E. Verlinde, M. Vonk, "On the partition sum of the NS fivebrane", hep-th/0205281.

[19] J. M. F. Labastida, C. Lozano, "Mathai-Quillen Formulation of Twisted N=4 Supersymmetric Gauge Theories in Four Dimensions", Nucl. Phys. B502 (1997) 741-790, hep-th/9702106.

[20] J. M. F. Labastida, C. Lozano, "Mass Perturbations in twisted N=4 Supersymmetric Gauge Theories", Nucl. Phys. B518 (1998) 37-58, hep-th/9711132.

[21] J.M.F. labastida, C. Lozano, "The Vafa-Witten Theory for Gauge Group SU(N)", Adv. Theor. Math. Phys. 3 (1999) 1201-1225, hep-th/9903172.

[22] J. M. F. Labastida, C. Lozano, "Duality in twisted $\mathrm{N}=4$ supersymmetric gauge theories in four dimensions", Nucl. Phys. B537 (1999) 203-242, hep-th/9806032.

[23] K. Yoshioka, "On Euler characteristic of moduli space of vector bundles", talk given in Kyoto Workshop on "Modular Invariance, ADE, Subfactors, and Geometry of Moduli Spaces", Nov 26-Dec 02 (2000).

[24] T. Eguchi, K. Sakai, "Seiberg-Witten Curve for the E-String Theory", hep-th/0203025.

[25] K. Mohri, "Exceptional String: Instanton Expansions and Seiberg-Witten Curve", hep-th/0110121. 
[26] S. Hosono, M. -H. Saito, A. Takahashi, "Holomorphic Anomaly Equation and BPS State Counting of Rational Elliptic Surface", Adv. Theor. Math. Phys. 3 (1999) 177-208, hep-th/9901151.

[27] R. Dijkgraaf, "The Mathematics of Fivebranes", hep-th/9810157.

[28] G. Bonelli, "The geometry of the M5-branes and TQFTs", J. Geom. Phys. 40 (2001) 13-25, hep-th/0012075.

[29] G. Bonelli, "On the Supersymmetric Index of the M-theory 5-brane and Little String Theory", Phys. Lett. B521 (2001) 383-390, hep-th/0107051.

[30] G. Bonelli, "The M5-brane on K3 and del Pezzos and multi-loo string amplitudes", JHEP 0112 (2001) 022, hep-th/0111126.

[31] A. Sen, B. Zwiebach, "Stable Non-BPS States in F-Theory", JHEP 03 (2000) 036, hep-th/9907164.

[32] T. Hauer, A. Iqbal, "Del Pezzo Surfaces and Affine 7-brane Backgrounds", JHEP 01 (2000) 043, hep-th/9910054.

[33] O. DeWolfe, T. Hauer, A. Iqbal, B. Zwiebach, "Uncovering Infinite Symmetries on [p,q] 7-branes: Kac-Moody Algebras and Beyond", Adv. Theor. Math. Phys. 3 (1999) 1785-1833, hep-th/9812028.

[34] T. Hauer, A. Iqbal, B. Zwiebach, "Duality and Weyl Symmetry of 7-brane Configurations", JHEP 0009 (2000) 042, hep-th/0002127.

[35] K. Yoshioka, "Euler characteristics of SU(2) instanton moduli spaces on rational elliptic surfaces", Commun. Math. Phys. 205 (1999) 501-517, math. AG/9805003.

[36] K. Yoshioka, "Twisted stability and Fourier-Mukai transform", math. AG/0106118.

[37] R. Gopakumar, C. Vafa, "M-Theory and Topological Strings-II", hep-th/9812127. 